\title{
IL-6 expression predicts treatment response and outcome in squamous cell carcinoma of the esophagus
}

Miao-Fen Chen ${ }^{1,2^{*}+}$, Ping-Tsung Chen ${ }^{3,4+}$, Ming Shian Lu ${ }^{5}$, Paul Yang Lin ${ }^{6}$, Wen-Cheng Chen ${ }^{1,2}$ and Kuan-Der Lee ${ }^{2,3}$

\begin{abstract}
Background: The identification of potential tumor markers can improve therapeutic planning and patient management. The aim of this study was to highlight the significance of IL-6 in esophageal squamous cell carcinoma (SCC).

Methods: We retrospectively analyzed the clinical outcomes of 173 patients with esophageal SCC, and examined the correlation between IL-6 levels and clinical outcomes in esophageal cancer patients. Furthermore, the human esophageal SCC cell line CE81T was selected for cellular and animal experiments to investigate changes in tumor behavior and treatment response after manipulation of IL-6 expression.

Results: In clinical outcome analysis, positive IL-6 staining and poor treatment response was significantly associated with shorter survival. Furthermore, the frequency of IL-6 immunoreactivity was significantly higher in esophageal cancer specimens than in non-malignant epithelium, and this staining was positively linked to the development of distant metastasis $(p=0.0003)$ and lower treatment response rates $(p=0.0001)$. By ELISA analysis, IL-6 serum levels were significantly elevated in patients developing disease failure.When IL-6 expression was inhibited, aggressive tumor behavior and radiation resistance could be overcome in vitro and in vivo. The underlying changes included increased cell death, less epithelial-mesenchymal transition and attenuated STAT3 activation. IL-6 inhibition was also associated with attenuated angiogenesis in tumor-bearing mice.
\end{abstract}

Conclusions: IL-6 was significantly associated with poor prognosis in patients with esophageal cancer. Targeting this cytokine could be a promising strategy for treatment of esophageal cancer, as evidenced by inhibition of aggressive tumor behavior and treatment resistance.

Keywords: IL-6, Esophageal squamous cell carcinoma, Prognosis

\section{Background}

Esophageal cancer, an aggressive upper gastrointestinal malignancy, generally presents as a locally advanced disease and requires a multimodal concept. Despite improvements in its detection and management, the prognosis in patients with esophageal cancer remains poor, with a 5 -year survival of $15-34 \%$ [1,2]. Most patients who undergo curative treatment for esophageal cancer will eventually relapse and die as a result of their disease. Neoadjuvant chemoradiotherapy (CCRT) can increase the

\footnotetext{
*Correspondence: miaofen@adm.cgmh.org.tw

${ }^{\dagger}$ Equal contributors

'Department of Radiation Oncology, Chang Gung Memorial Hospital at Chiayi, Chiayi, Taiwan

${ }^{2}$ Chang Gung University, College of Medicine, Taoyuan, Taiwan

Full list of author information is available at the end of the article
}

chance of R0 resection, and responders will have a survival benefit [3]. Patient selection is important to guide multidisciplinary therapy. The identification of potential molecular markers to predict response to CCRT and recurrence is important for the effective management and prognosis of esophageal cancer.

Proinflammatory cytokine may contribute to tumor progression by stimulating angiogenesis, invasion and metastasis $[4,5]$ IL-6 is a pleiotropic cytokine that is capable of modulating diverse cell functions such as inflammatory reactions, and is a major activator of the JAK/STAT3 and PI3K/AKT signaling pathways [6]. IL-6 signaling has been implicated in the regulation of tumor growth and metastatic spread in different cancers [7-9]. Moreover, increased IL-6 serum levels were reported to

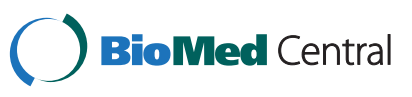


be associated with metastasis and poor prognosis in prostate, ovarian and gastrointestinal cancers [10-12]. Although evidence suggests that IL-6 is a critical factor in a variety of malignancies $[11,13,14]$, how IL- 6 modulates the biological activities of esophageal carcinoma cells and how it is associated with the prognosis of esophageal cancer remains unclear. There are two distinct histological types of esophageal cancer: squamous cell carcinoma (SCC) and adenocarcinoma. There are marked differences between these carcinomas in incidence, natural history and treatment outcomes [15]. The majority of esophageal SCC cases occur in Asia, with the predominant type in Taiwan being SCC [16]. We therefore investigated the role of IL-6 in esophageal SCC in vitro and in vivo, and examined the correlation between IL- 6 levels and clinical outcomes in esophageal cancer patients.

\section{Materials and methods}

\section{Patient characteristics}

The Institutional Review Board of Chang Gung Memorial Hospital approved the present study (Permit Number: 96-1693B). The written consents were signed by the patients for their specimen and information to be stored in the hospital and used for research. Patients who did not comply with the treatment regimen and those who received surgery alone for early-stage esophageal cancer were excluded from the study. A total of 173 patients with esophageal SCC who completed curative treatment were enrolled in the study. The curative treatment for esophageal cancer included neoadjuvant CCRT combined with surgery or definitive CCRT according to the guidelines proposed by oncology team at our hospital. On completion of neoadjuvant CCRT, patients underwent a repeat CT scan and endoscopic examination to determine the response to treatment. If the tumor was considered resectable, patients underwent surgery for the residual tumor. Pathologic complete response (CR) was defined as absence of residual invasive tumor in the surgical specimen in patients undergoing surgical intervention. For patients who refused surgery or in whom it was contraindicated, a second round of CCRT was administered, comprising two courses of chemotherapy and radiotherapy of a total of 60-63 Gy. Specimens collected from the 173 patients at diagnosis were subjected to immunochemical analysis. The main end points were overall survival (OS), disease-free survival (DFS) and

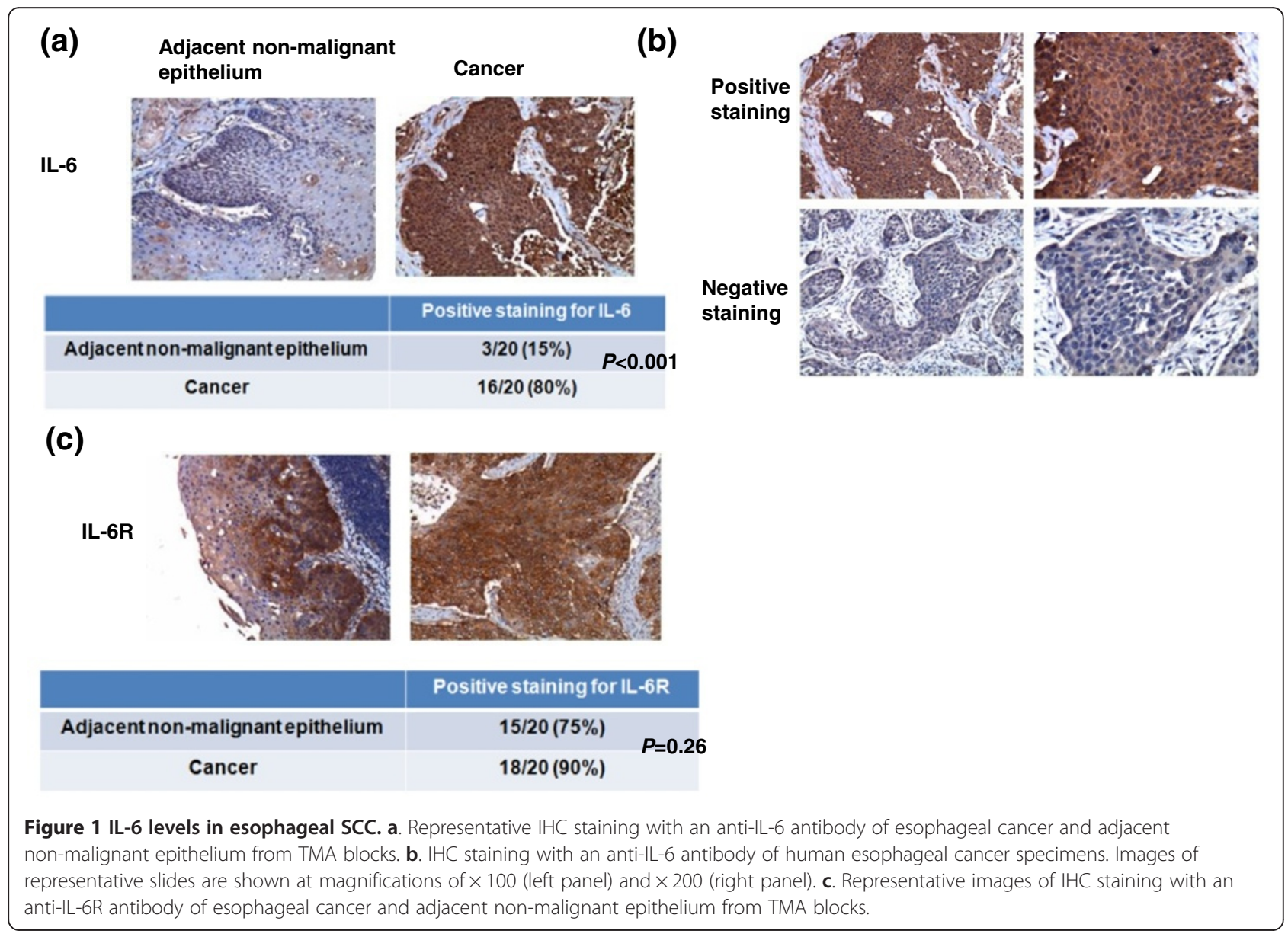


response to neoadjuvant therapy. Survival probability was analyzed statistically using the Kaplan-Meier method. The significance of between-group differences was assessed using the log-rank test. Multivariate analyses were performed using a Cox regression model for survival.

\section{Immunohistochemical staining (IHC)}

Formalin-fixed, paraffin-embedded tissues from 173 patients with esophageal cancer were subjected to IHC staining. Dissected esophageal cancer specimens from 20 of these patients were converted into tissue microarray (TMA) blocks using an AutoTiss 1000 arrayer (Ever BioTechnology, Canada). The TMA block contained esophageal SCCs and the adjacent non-malignant epithelium. The quality of the TMA slides was confirmed by the pathologist using hematoxylin- and eosin-stained slides. The IHC data for the specimens were assessed using the semiquantitative immunoreactive score (IRS). The criterion for positive staining was an IRS score of $\geq 2$. The details were described in Additional file 1: Supplementary methods.

\section{Cell culture and reagents}

The human esophageal cancer cell line CE81T, derived from a well-differentiated esophageal SCC, was obtained from the Bioresource Collection and Research Center (BCRC), and cultured in Dulbecco's modified Eagle's medium supplemented with $10 \%$ fetal bovine serum. The IL-6-neutralizing antibody, STAT3 siRNA and the JAK inhibitor AG490 were purchased from R\&D Systems (Minneapolis, MN) and Sigma (St. Louis, MO), respectively. The IL-6-GFP silencing vector (human IL6 shRNA constructs in retroviral GFP vector) and GFP-control vector (Non-effective scrambled shRNA cassette in retroviral GFP vector) were purchased from Origene Technologies, Inc. (Rockville, MD). Transfection in CE81T was carried out by Lipofectamine ${ }^{\mathrm{TM}} 2000$ (Invitrogen) according to the manufacturer's instructions. Stable cancer cells were generated by transfecting CE81T cells with either the IL-6 silencing vectors or control vectors, followed by selection with puromycin for 4 weeks.

\section{Cell growth}

To measure cell growth, $1 \times 10^{4}$ cells per well were plated into 6-well dishes. At the indicated time-points, cells were trypsinized, collected and surviving cells counted using Trypan blue exclusion, from which the survival curves for CE81T transfectants ( wild type, with control vectors, and with IL-6 silencing vectors) were established.

\section{Immunoblotting}

To determine the in vitro effects of STAT3 siRNA, proteins were extracted from cells transfected with STAT3 siRNA for $72 \mathrm{~h}$. To determine the in vitro effects of the JAK inhibitor and the anti-IL- 6 antibody, proteins were extracted from cells incubated in the presence of $50 \mu \mathrm{M}$ AG490 or $5 \mu \mathrm{g} / \mathrm{ml}$ IL-6 neutralizing antibodies for $24 \mathrm{~h}$. The details were described in Additional file 1: Supplementary methods.

Immunofluorescence staining (IF) and Statistical analysis The details were described in Additional file 1: Supplementary methods.

\section{Enzyme-linked immunosorbent assay (ELISA) analysis of IL-6 level}

We examined the serum level of IL-6 from 71 patients among subjects enrolled with esophageal cancer in the

Table 1 Clinico-pathological characteristics of esophageal cancer patients for immunohistochemical investigation

\begin{tabular}{|c|c|c|c|}
\hline & \multicolumn{2}{|c|}{ No. of patients } & \multirow[b]{2}{*}{$p$ value } \\
\hline & IHC-IL-6(-) & IHC- IL-6 (+) & \\
\hline patients & 85 & 88 & \\
\hline \multicolumn{4}{|l|}{ Age } \\
\hline$>=56 y / 0$ & 43 & 44 & 0.938 \\
\hline$<56 y / 0$ & 42 & 44 & \\
\hline \multicolumn{4}{|l|}{ Location } \\
\hline Upper third & 22 & 26 & 0.498 \\
\hline Middle third & 48 & 42 & \\
\hline Lower third & 15 & 20 & \\
\hline Tumor stage & & & $0.041^{*}$ \\
\hline$|-| \mid$ & 28 & 17 & \\
\hline III-IV & 57 & 71 & \\
\hline LN metastasis & & & 0.246 \\
\hline negative & 27 & 21 & \\
\hline positive & 58 & 67 & \\
\hline P-stat3 staining & & & $<0.0001^{*}$ \\
\hline negative & 71 & 20 & \\
\hline positive & 14 & 68 & \\
\hline Response to Neoadjuvant Tx & & & $0.0001^{*}$ \\
\hline Response & 69 & 47 & \\
\hline Non- response & 16 & 41 & \\
\hline Surgery s/p Neoadjuvant Tx & & & 0.145 \\
\hline Yes & 31 & 24 & \\
\hline No & 54 & 64 & \\
\hline $\begin{array}{l}\text { Local-regional } \\
\text { Recurrence /persistent }\end{array}$ & & & $<0.0001^{*}$ \\
\hline No & 44 & 16 & \\
\hline Yes & 41 & 72 & \\
\hline Distant metastasis & & & $0.0003^{*}$ \\
\hline negative & 58 & 36 & \\
\hline positive & 27 & 52 & \\
\hline
\end{tabular}

Abbreviations: Neoadjuvant Tx = neoadjuvant chemoradiotherapy. 
present study. For serum specimen, five milliliters of peripheral blood were drawn from each patient. Moreover, IL-6 levels in cellular supertnant and murine serum were tested. Levels of IL-6 in samples were analyzed using an IL-6 Quantikine ELISA Kit (R\&D system). The details were described in Additional file 1: Supplementary methods.

\section{Tumor xenografts}

This study was carried out in strict accordance with the recommendations in the Guide for the Care and Use of Laboratory Animals as promulgated by the Institutes of Laboratory Animal Resources, National Research Council, U.S.A. The protocol was approved by the Committee on the Ethics of Animal Experiments of Chang Gung
Memorial Hospital (Permit Number: 2012080903). Eight-week-old male athymic nude mice were used and all animal experiments conformed to the protocols approved by the experimental animal committee of our hospital. CE81T cancer cell transfectants $\left(1 \times 10^{6}\right.$ per implantation, five animals per group) were subcutaneously implanted in the dorsal gluteal region. To determine in vivo radiosensitivity, local irradiation (15 Gy) was performed when the tumor volume reached $500 \mathrm{~mm}^{3}$. Radiosensitivity was indicated by a growth delay (i.e., the time required for the tumor to recover its previous volume after irradiation). Duplicate experiments were performed for growth delay analyses. The details were described in Additional file 1: Supplementary methods.

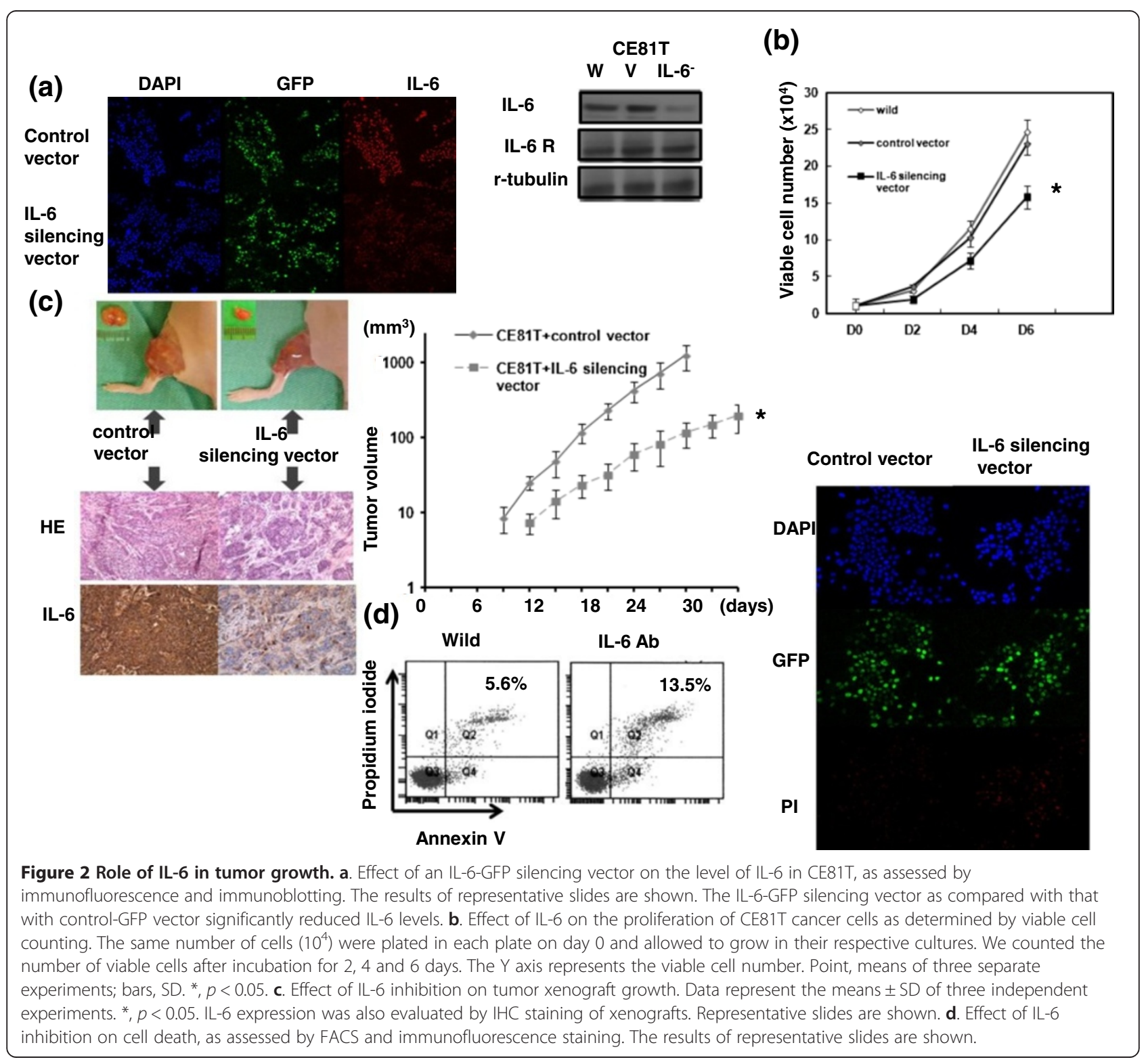



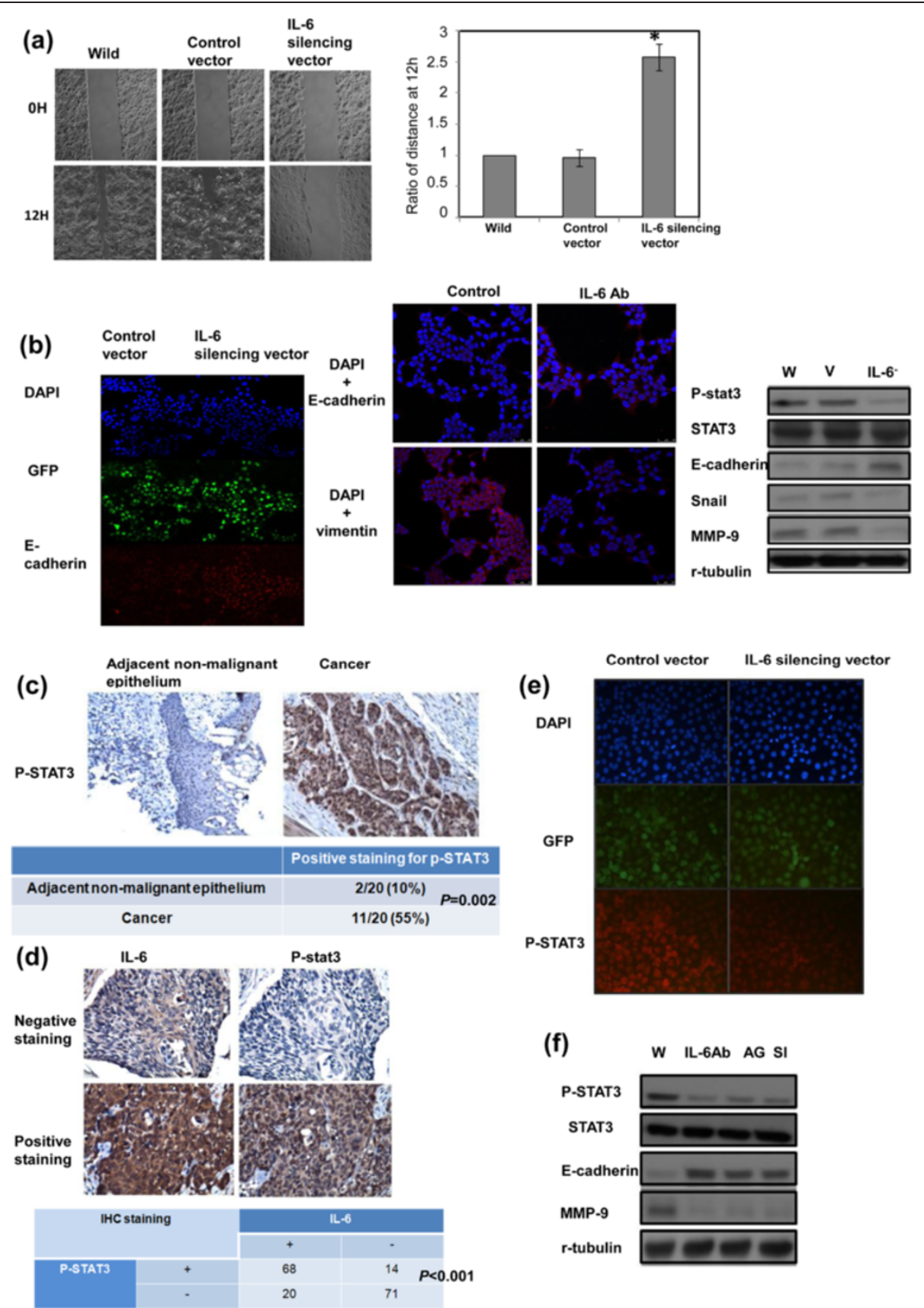

Figure 3 Role of IL- 6 in aggressive tumor behavior and EMT changes. a. Effect of IL-6 inhibition on the migration ability of esophageal cancer cells. Plates were photographed at the times indicated. Representative slides and quantitative data (y-axis shows the relative ratio, normalized to the distance under control conditions) are shown. $\mathbf{b}$. Effect of IL-6 inhibition on EMT-associated proteins. The changes of Ecadherin and vimentin expression were evaluated by immunofluorescence, and the results of representative slides are shown. In addition, changes in the levels of EMT-associated proteins were evaluated by immunoblotting (W, wild-type cells; $V$, cells transfected with the control vector; IL-6, cells transfected with the IL-6 silencing vector). c. Representative IHC staining with an anti-p-STAT3 antibody in esophageal cancer and adjacent non-malignant epithelium on slides from TMA blocks. d. P-STAT3 levels correlate positively with IL-6 levels in human esophageal cancer specimens $(p<0.001)$. Representative images of positive IL- 6 and p-STAT3 staining on slides from a selected tumor specimen, and representative negative staining for IL-6 and p-STAT3 on slides from another tumor specimen, are shown. e. Effect of the IL-6 silencing vector on STAT3 activation, as examined by immunofluorescence analysis. f. Effect of inhibited IL-6 signaling on STAT3 activation and EMT-related protein levels, as determined by immunoblotting (W, proteins were extracted from cells under control condition; IL-6Ab, proteins were extracted from cells incubated in the presence of $5 \mathrm{\mu g} / \mathrm{ml} \mathrm{IL-6}$ neutralizing antibodies for $24 \mathrm{~h} ; \mathrm{AG}$, proteins were extracted from cells incubated in the presence of $50 \mu \mathrm{M}$ AG490 for $24 \mathrm{~h}$; Sl, proteins were extracted from cells $72 \mathrm{~h}$ after transfection with STAT3 siRNA). 


\section{Results}

Level of IL-6 in esophageal SCC

The IHC data for TMA slides demonstrated that IL-6 was overexpressed in tumor tissues compared to adjacent non-malignant epithelial tissues (Figure 1a). Figure $1 \mathrm{~b}$ showed the representative slides of positive staining and negative staining with anti-IL-6 antibody for human esophageal cancer specimens. Of the 173 esophageal cancer tissues, 88 (51\%) showed positive IL-6 immunoreactivity $(41 \%(45 / 109) \quad$ in $\leq \mathrm{T} 3$ versus $67 \%(43 / 64)$ in T4, $P=0.001)$. Furthermore, there was a positive correlation between IL-6 overexpression and cancers developing loco-regional failure or distant metastasis (Table 1). The biological activities of IL-6 are mediated through binding to a membrane-bound or soluble form of IL-6 receptor. By IHC analysis of TMA slides, IL-6 receptor (IL-6R) was expressed in both tumor tissues and adjacent non-malignant epithelial tissues (Figure 1c).

\section{Role of IL-6 in tumor growth}

As demonstrated in Figure 2a, the IL-6 silencing vector significantly inhibited IL-6 expression in CE81T cells. By viable cell counting over 6 days and observation of xenograft tumors, the IL-6 silencing vector significantly inhibited tumor growth in vitro (Figure $2 \mathrm{~b}$ ) and in vivo (Figure 2c). Figure $2 \mathrm{~d}$ showed that the cell death rate increased from $5.6 \pm 1.2 \%$ to $13.5 \pm 1.8 \%$ in CE81T cells after treatment with an IL-6-neutralizing antibody (as measured by flow cytometry) and by the IL- 6 silencing vector (propidium iodide staining).

\section{Role of IL-6 in tumor invasion and underlying mechanisms}

There was a positive link between IL- 6 and tumor stage and disease failure in patients with esophageal cancer (Table 1). Furthermore, as demonstrated using migration scratch assays, IL-6 silencing vector attenuated the (a)

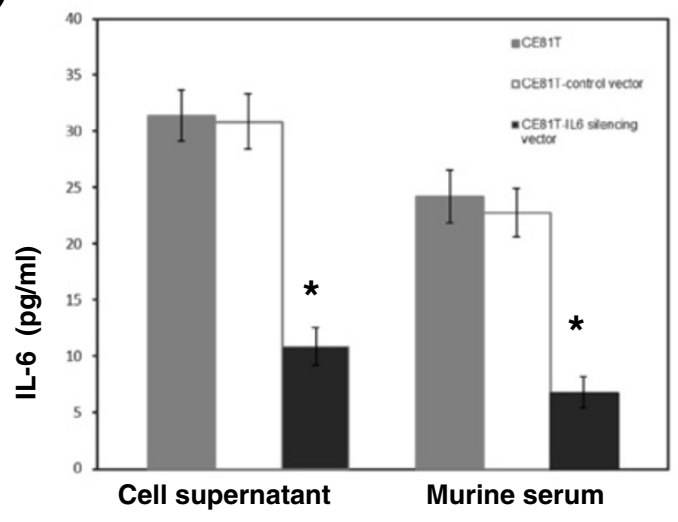

(b)
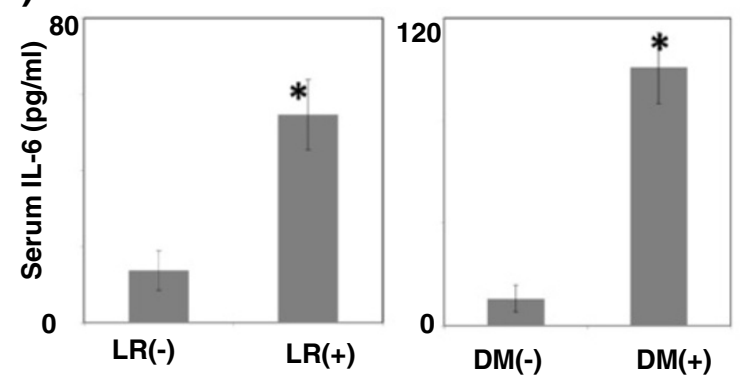

\section{(c) 20 days after tumor implantation}
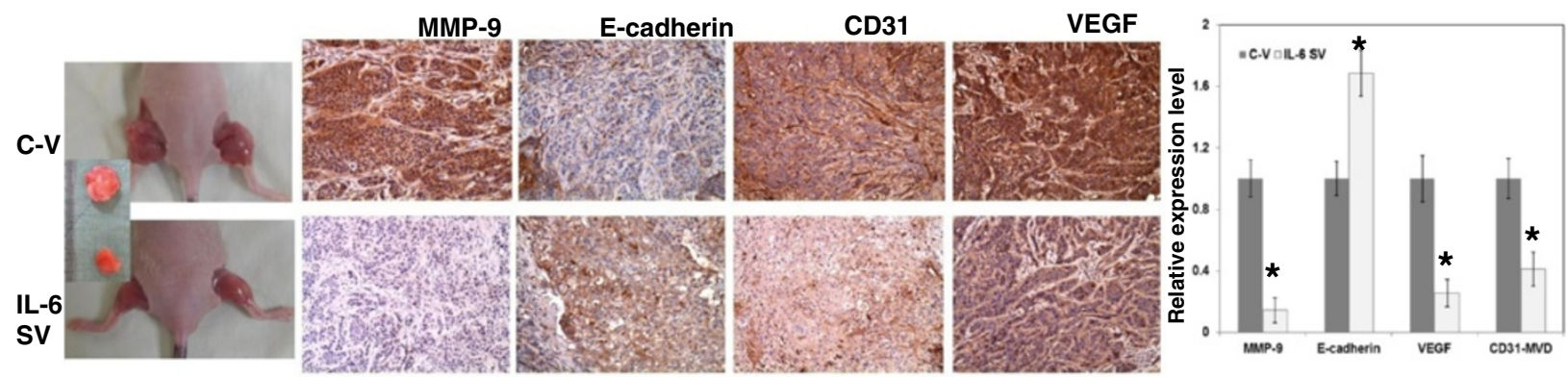

Figure 4 Effects of circulating IL- 6 on tumor aggressiveness. a. The levels of IL-6 in cell supernatant and serum of mice bearing tumors with or without IL-6 silencing vectors were examined by ELISA. Columns, means of 3 separate experiments; bars, SD. *, P<0.05. b. Serum IL-6 levels of patients were examined by ELISA analysis. Columns are the means of specimen; Bars, SD; ${ }^{*}, P<0.05$. (LR= Local-regional Recurrence /persistent; DM = distant metastasis). c. IHC using MMP-9, E-cadherin, CD31, and VEGF staining in tumors 20 days after implantation. IL-6 silencing vectors were significantly reduced by the angiogenesis and EMT-related changes as compared with control-GFP vector. The Y-axis represents the ratio normalized by the value of target protein in tumor transfected with control vectors. Columns, means of 3 separate experiments; bars, SD. *, $P<0.05$ (C-V, cells transfected with the control vector; IL-6 SV, cells transfected with the IL-6 silencing vector). 
invasive capacity of esophageal cancer cells (Figure 3a). Epithelial-mesenchymal transition (EMT) is a key event in invasiveness [17]. As shown in Figure 3b, the IL-6 silencing vector reversed EMT changes, with increased E-cadherin expressions, and decreased matrix metalloproteinase (MMP)-9, vimentin and Snail, a repressor of E-cadherin expression. The activation of STAT3 signaling was reported to play important roles in the induction of aggressive tumor behavior and EMT changes in cancer [18], including cancers in the upper aerodigestive tract $[8,19]$. As shown in Table 1 and Figure $3 c-d$, positive staining for p-STAT3 was evident in $47 \%$ of the 173 cancer specimens, and a significant positive correlation was found in cancer specimen that expressed IL-6 and p-STAT3. As determined by immunofluorescence and immunoblotting analysis (Figure 3e-f), IL-6 inhibition significantly attenuated the activation of STAT3 in vitro. Moreover, when STAT3 signaling was inhibited by STAT3 siRNA or the JAK inhibitor AG490, the decreases in EMT- related protein levels were comparable to those induced by the IL-6-neutralizing antibody (Figure 3f). Therefore, it appears that altered STAT3 activation and subsequent EMT might, at least in part, be responsible for the aggressive tumor behavior in IL-6-positive esophageal cancer.

\section{Effects of circulating IL- 6 on tumor aggressiveness}

We used ELISA assay to test the level of IL-6 in the supernatant of cell culture and the serum of mice bearing tumors. As shown in Figure 4a, IL-6 silencing vector clearly attenuated IL-6 secretion. Moreover, the serum levels of IL- 6 were examined by ELISA. The mean IL- 6 levels in serum samples from 71 patients with esophageal cancer were $39 \pm 7.7 \mathrm{pg} / \mathrm{mL}$. Serum IL-6 levels were significantly elevated in patients with local-regional failure or developing distant metastasis compared to those in patients with disease control (Figure 4b). Table 2 also showed that IL-6 serum levels were significantly correlated with a greater probability of developing local-regional failure and distant metastasis. In addition to EMT, angiogenesis is one of the mechanisms that promote tumor progression. The most prominent and best-characterized pro-angiogenic pathway is vascular endothelial growth factor (VEGF) signaling [20], and CD31-mediated endothelial cell-cell interactions are involved in angiogenesis [21]. Therefore, the vascular network within the tumor was measured by VEGF staining and microvascular density (MVD) analysis after CD31 staining. When esophageal cancer cells with control vectors and those with IL-6 silencing vectors were subcutaneously implanted into mice, we found that the growth inhibiting effect induced by IL-6 silencing vector associated with lower expression levels of EMT- and angiogenesis-related factor (Figure 4c). Accordingly, we suggested that circulating IL-6 plays a
Table 2 Significance of IL-6 expression stratified according to the occurrence of local- regional failure (LR) or distant metastasis (DM) in 71 patients

\begin{tabular}{lcccc}
\hline & & \multicolumn{2}{c}{ IL-6 serum level } & \\
\cline { 3 - 4 } & & $<$ mean & $>=$ mean & \\
\hline LR & + & 12 & 32 & $P=0.001$ \\
& - & 18 & 9 & \\
DM & + & 10 & 24 & $P=0.035$ \\
& - & 20 & 17 & \\
\hline
\end{tabular}

$\mathrm{LR}=$ Local-regional Recurrence /persistent; $\mathrm{DM}=$ distant metastasis.

role in tumor promotion, and the induction of angiogenesis and EMT might be the underling mechanisms.

\section{IL-6 correlates with treatment response in esophageal SCC}

Regarding clinical data, the expression of IL-6 was significantly associated with a lower rate of response to curative treatment in the 173 esophageal SCC patients (Table 1), and a lower pathological complete response rate in the 55 patients who underwent surgical intervention (Table 3). Therefore, we investigated the role of IL-6 in treatment resistance and the underlying mechanisms. Colony-forming assay data (Figure 5a) and the in vivo delay in tumor growth (Figure $5 \mathrm{~b}$ ) demonstrated that the IL-6 silencing vector significantly sensitized esophageal cancer cells to irradiation. As shown in Figure $5 \mathrm{c}-\mathrm{d}$, inhibition of IL- 6 increased cell death and DNA damage and attenuated STAT3 activation after irradiation. Tumor vascularity have been shown to be linked to the tumor-bed effect induced by irradiation [22]. To investigate whether angiogenesis underlies radiation resistance triggered by IL-6, the vascular network within the tumor by MVD analysis and the expression of VEGF after irradiation were measured. As shown in Figure $5 d$, the IL-6 silencing vector significantly decreased angiogenesis by evidence of decreased MVD and VEGF staining in irradiated tumors.

\section{Correlation between the IL- 6 level and clinical outcome}

As shown in Figure 6, IL-6 is a significant predictor for OS. The median OS times were 16 and 42 months in patients who completed CCRT treatment and those who underwent surgical intervention, respectively. In addition, Tables 1, 2, 3 showed that IL-6 was significantly correlated

Table 3 Significance of IL-6 expression stratified according to the complete response to neoadjuvant treatment in $\mathbf{5 5}$ patients with surgery resection

\begin{tabular}{lcccc}
\hline & & \multicolumn{3}{c}{ IL-6 } \\
\cline { 3 - 5 } & & + & - & \\
\hline Pathologic CR & + & 3 & 11 & $P=0.052$ \\
& - & 21 & 20 & \\
\hline
\end{tabular}




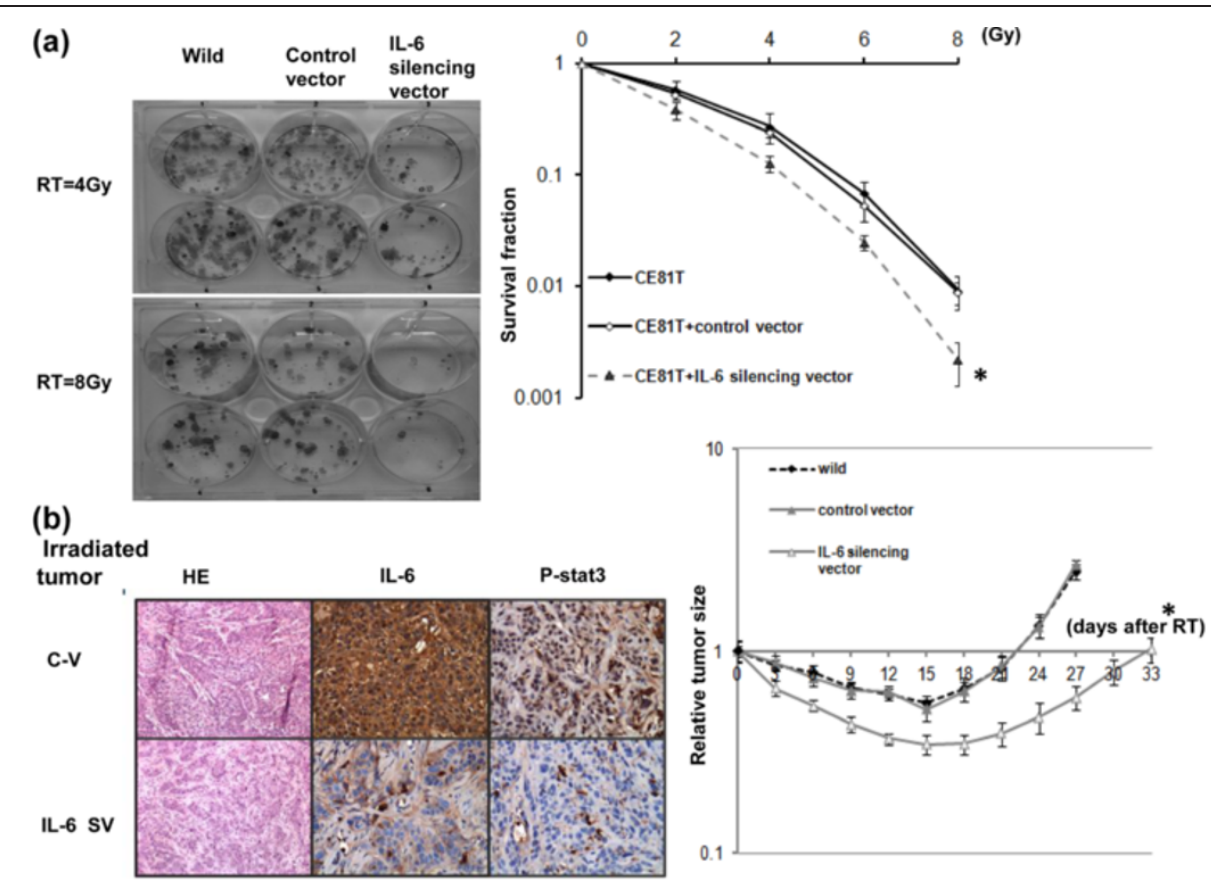

(c)

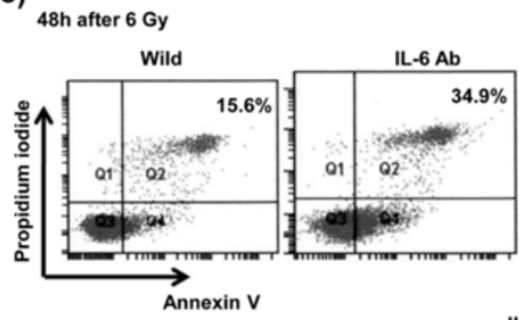

(d) 14 days after RT

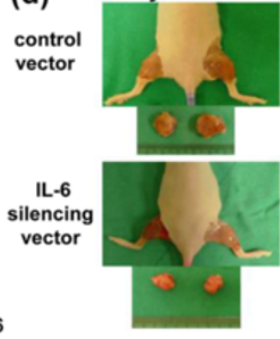

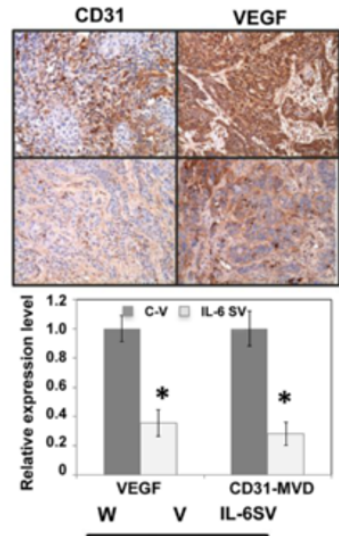

IL-6

VEGF

CD31

P-STAT3

STAT3

r-tubulin

P.H2AX

$\operatorname{lamin} \beta 1$

Figure 5 Effects of IL-6 on radiation responses. a. Cells were irradiated with 0, 2, 4, 6, and 8 Gy, and survival curves were constructed using colony-forming assay data. Data represent the means of three experiments. ${ }^{*}, p<0.05$. IL-6 silencing vectors significantly sensitized tumor cells to irradiation. $\mathbf{b}$. Effects of IL-6 on in vivo radiosensitivity, as assessed by evaluation of tumor growth following irradiation (15 Gy). The y-axis shows the tumor volume ratio at each time point, divided by the tumor volume at irradiation. (C-V, cells transfected with the control vector; IL-6 SV, cells transfected with the IL-6 silencing vector). $\mathbf{c}$. The in vitro effects of IL-6 inhibition on p-STAT3 and p-H2AX levels and cell death, as evaluated by IHC staining and Annexin V-PI staining in irradiated cells. $\mathbf{d}$. An IL-6-silencing vector decreased angiogenesis in tumors, as evaluated by immunoblotting and $\mathrm{IHC}$ analysis of tumor specimens 14 days after local irradiation. Representative slides are shown. The $\mathrm{Y}$ axis represents the ratio normalized by the value of target protein in tumor transfected with control vectors. Columns, means of 3 separate experiments; bars, SD. * , $P<0.05$. (C-V, cells transfected with the control vector; IL-6 SV, cells transfected with the IL-6 silencing vector). 
with a greater probability of developing distant metastasis and a higher recurrence rate after curative treatment. In univariate and multivariate analyses, poor treatment response, no tumor resection, and positive IL-6 staining were significantly associated with shorter survival (Tables 4, 5, 6). Furthermore, in the subgroup of 118 patients treated with CCRT, but not in the 55 who underwent surgical intervention, positive IL-6 staining retained predictive power concerning survival in a multivariate analysis (Tables 7,8 ).

\section{Discussion}

Understanding the molecular mechanisms underlying aggressive tumor growth in esophageal SCC is pivotal to identifying novel targets for pharmacological intervention. Furthermore, clinical data $[2,3,23]$ suggest the need for markers that predict responses to neoadjuvant CCRT and help to identify patients at high risk of tumor recurrence and distant metastasis. However, no suitable markers have been identified to date. The inflammatory cytokine IL-6 contributes to the growth and progression of various malignancies, such as HNSCC, prostate cancer and gastrointestinal cancer, and acts as an autocrine growth factor and an anti-apoptotic factor for various stimuli, including anticancer agents [11,24-27]. Moreover, the IL-6 /STAT3 pathway is important for the development of inflammation-associated intestinal tumorigenesis. Similar to other series $[11,28]$, we found that IL- 6 and IL-6R were detected in both esophageal cancer specimens and esophageal carcinoma cell lines. IL-6 expression was higher in esophageal cancer specimens compared with non-malignant tissues. Furthermore, positive staining for IL-6 was associated with higher tumor stage, higher rates of tumor recurrence and distant metastasis. To further investigate whether IL-6 was responsible for aggressive tumor growth in esophageal SCC, we suppressed IL-6 in esophageal cancer cells using a silencing vector. Data obtained from cell and xenograft tumor growth experiments revealed that inhibiting IL-6 resulted in slower tumor growth and reduced invasiveness. The transformation of an epithelial cell into a mesenchymal cell appears to be functionally relevant to the invasive characteristics of epithelial tumors, including esophageal cancer $[19,29,30]$. At the molecular marker level, EMT is characterized by the loss of E-cadherin and increased expression of invasion-related factors [31]. In cell experiments, the IL-6 silencing vector induced both an increase in E-cadherin levels in esophageal cancer cells, and decreases in MMP-9 levels. Constitutive activation of STAT3 signaling has been reported to contribute to oncogenesis by promoting proliferation and EMT, and IL-6 is a major activator of JAK/STAT3 signaling [8,32-34]. Therefore, we examined the links between STAT3 activation, IL- 6 and EMT. When blocking STAT3

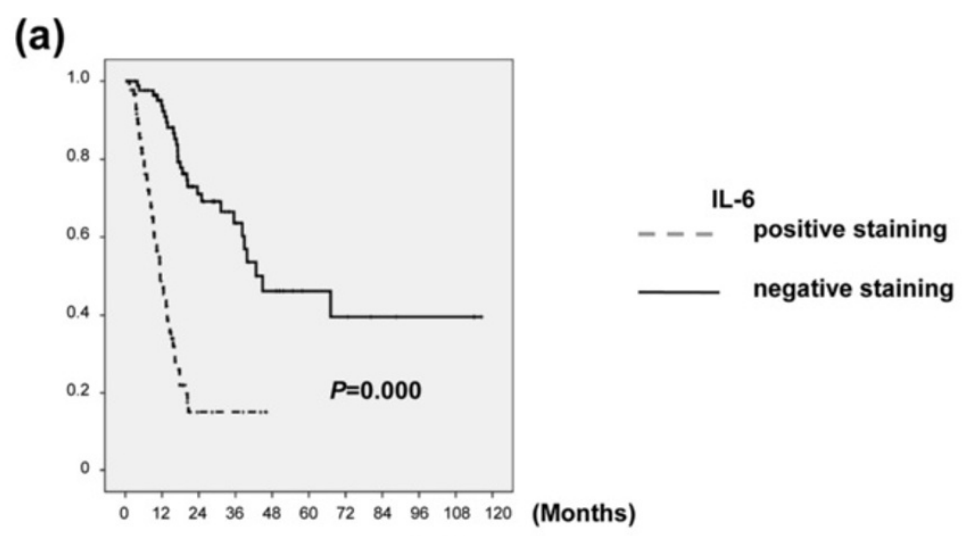

(b)

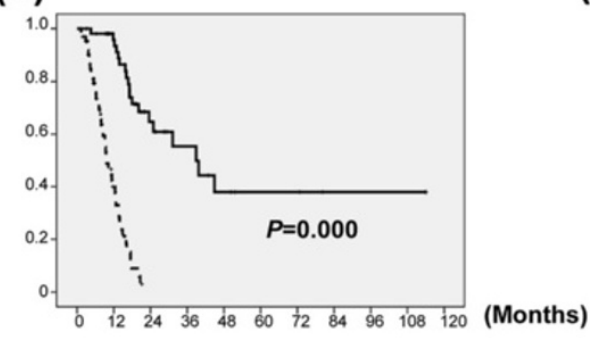

(c)

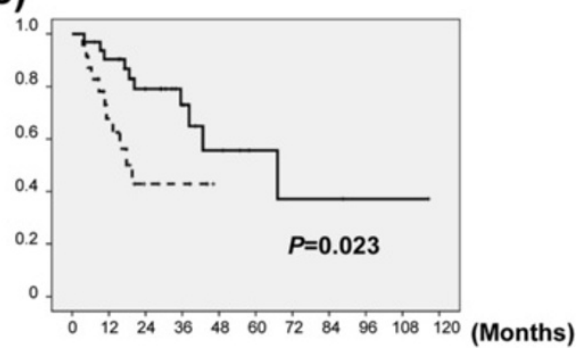

Figure 6 Correlation between IL-6 level and clinical outcome. Survival differences according to IL-6 positivity for (a) all 173 patients, (b) patients treated with CCRT and (c) patients who underwent pre-operative CCRT and surgery. The IL-6-positive group exhibited shorter survival than the IL-6-negative group. 
Table 4 Univariate analysis to determine factors associated with prognosis

\begin{tabular}{lcc}
\hline Variables & $\begin{array}{c}\text { P value for } \\
\text { overall survival }\end{array}$ & $\begin{array}{c}\text { P value for } \\
\text { disease-free survival }\end{array}$ \\
\hline Clinical stage & 0.271 & $0.006^{*}$ \\
Tumor resection & $0.001^{*}$ & $0.000^{*}$ \\
Positive staining for IL-6 & $0.000^{*}$ & $0.000^{*}$ \\
Positive staining for p-STAT3 & $0.000^{*}$ & $0.000^{*}$ \\
Local-regional Recurrence & $0.002^{*}$ & $0.000^{*}$ \\
Distant metastasis & $0.03^{*}$ & $0.000^{*}$ \\
Treatment response & $0.000^{*}$ & $0.000^{*}$ \\
\hline
\end{tabular}

activation by STAT3 siRNA or JAK inhibitor, the decreases in EMT-related protein levels were comparable to those induced by IL-6 silencing vector. Furthermore, IHC data obtained from clinical samples demonstrated that IL-6 expression was significantly correlated with p-STAT3 staining. Therefore, it is likely that STAT3 activation plays a role in transmitting IL-6 signals to downstream targets that regulate EMT and invasiveness.

In the present study, IL-6 silencing vectors significantly decreased IL-6 levels seen in the supernatant of cell culture medium and the serum of mice bearing tumors. Moreover, in the clinic, IL-6 serum levels seem to be elevated in a subgroup of patients with local-regional failure or distant metastasis. We assume that circulating IL-6 plays an important role to stimulate tumor growth in vivo, besides direct action on malignant cells. Angiogenesis is one of the mechanisms that promote tumor progression, and CD31-mediated endothelial cell-cell interactions are involved in angiogenesis. Moreover, STAT3 activation has been reported to modulate the expression of genes that mediate angiogenesis; e.g., VEGF [35]. Accordingly, the links between IL-6, angiogenesis, and promotion of cancer in tumor-bearing mice were further investigated using a xenograft tumor model. Our data from animal experiments demonstrated that IL-6 level positively linked with angiogenesis in addition to EMT. These findings indicated that the promotion of EMT changes and

Table 5 Multivariate analysis to determine molecular markers associated with prognosis (OS) of patients

\begin{tabular}{lccl}
\hline Variables & $\begin{array}{c}\text { Odd } \\
\text { ratios }\end{array}$ & $\begin{array}{c}\text { 95\% confidence } \\
\text { interval }\end{array}$ & p \\
\hline IL-6 staining & 5.319 & $2.906-9.734$ & $<0.001^{*}$ \\
P-STAT3 staining & 0.926 & $0.529-1.593$ & 0.761 \\
Tumor resection & 0.454 & $0.270-0.762$ & $0.003^{*}$ \\
Clinical stage & 0.908 & $0.531-1.551$ & 0.723 \\
Treatment response & 2.601 & $1.583-4.276$ & $<0.001^{*}$ \\
Recurrence and/or distant metastasis & 4.172 & $1.700-10.240$ & $0.002^{*}$ \\
\hline
\end{tabular}

Table 6 Multivariate analysis to determine molecular markers associated with prognosis (DFS) of patients

\begin{tabular}{lccc}
\hline Variables & Odd ratios & 95\% confidence interval & $\mathbf{p}$ \\
\hline IL-6 staining & 5.424 & $3.349-8.784$ & $<0.001^{*}$ \\
p-STAT3 staining & 0.724 & $0.465-1.128$ & 0.153 \\
Tumor resection & 0.365 & $0.241-0.553$ & $<0.001^{*}$ \\
Clinical stage & 1.655 & $1.058-2.589$ & $0.027^{*}$ \\
Treatment response & 3.719 & $2.135-4.733$ & $<0.001^{*}$ \\
\hline
\end{tabular}

angiogenesis mediate the aggressive tumor growth noted in IL-6 expressing esophageal cancer, at least in part.

Radiotherapy is a well-established therapeutic modality in oncology. It provides survival benefits in several cancer types, including esophageal cancer. For esophageal SCC, treatment response is an independent prognostic factor. We demonstrated that positive IL-6 staining was significantly associated with a lower response rate after treatment in patients with esophageal SCC. As determined using clonogenic assays and delayed tumor growth, inhibition of IL- 6 by transfection of an IL- 6 silencing vector increased radiosensitivity. Extensive DNA damage caused by radiation and anti-cancer agents can result in cell death or sensitivity to clinical treatment if left unrepaired [36]. Phosphorylated H2AX is an indicator of double-strand breaks, and its expression after irradiation correlates with treatment sensitivity [37]. Our data demonstrate that inhibition of IL- 6 was associated with increased radiation-induced DNA damage and increased cell death. Moreover, the data from xenograft tumors demonstrated that inhibition of IL- 6 attenuated angiogenesis and decreased p-STAT3 activation after irradiation. The expression of angiogenic factors is suggested to have predictive value for treatment response and outcome in patients with esophageal cancer [38,39]. Therefore, we suggest activation of STAT3 signaling and angiogenesis after irradiation was reported to be responsible for resistance to treatment and tumor regrowth after irradiation triggered by IL- 6 .

We further examined the predictive power of IL-6 for prognosis in esophageal SCC. Besides a lower response to

Table 7 Multivariate analysis to determine factors associated with prognosis (OS) of patients with definite CCRT treatment

\begin{tabular}{lccc}
\hline Variables & $\begin{array}{c}\text { Odd } \\
\text { ratios }\end{array}$ & $\begin{array}{c}\mathbf{9 5 \%} \text { confidence } \\
\text { interval }\end{array}$ & $\mathbf{p}$ \\
\hline IL-6 staining & 7.622 & $3.580-16.226$ & $<0.001^{*}$ \\
p-STAT3 staining & 1.032 & $0.524-2.031$ & 0.928 \\
Clinical stage & 0.828 & $0.409-1.677$ & 0.600 \\
Treatment response & 2.327 & $1.314-4.120$ & $0.004^{*}$ \\
Recurrence and/or distant metastasis & 4.576 & $1.292-16.199$ & $0.018^{*}$ \\
\hline
\end{tabular}


Table 8 Multivariate analysis to determine factors associated with prognosis (OS) of patients with pre-op CCRT and surgery

\begin{tabular}{lccc}
\hline Variables & $\begin{array}{c}\text { Odd } \\
\text { ratios }\end{array}$ & $\begin{array}{c}\text { 95\% confidence } \\
\text { interval }\end{array}$ & $\mathbf{p}$ \\
\hline IL-6 staining & 1.738 & $0.537-5.622$ & 0.356 \\
P-STAT3 staining & 1.152 & $0.364-3.643$ & 0.810 \\
Clinical stage & 0.860 & $0.332-2.232$ & 0.757 \\
Pathologic CR & 1.883 & $0.518-6.842$ & 0.336 \\
Recurrence and/or distant metastasis & 4.205 & $1.138-15.538$ & $0.031^{*}$ \\
\hline
\end{tabular}

treatment, enhanced expression of IL-6 was significantly associated with a higher clinical stage, a greater risk of distant metastasis and shorter survival. In a multivariate analysis, IL-6 retained predictive power for OS.

These findings indicate that IL-6-positive esophageal cancer provides a suitable microenvironment for the development of tumor growth and treatment resistance mediated by induction of angiogenesis, enhancement of cell mobility, and promotion of EMT. Our data support the emerging notion that IL-6 and p-STAT3 are clinically significant predictors, and suggest that IL-6 may represent a suitable target for esophageal SCC treatment.

\section{Additional file}

Additional file 1: Supplementary methods.

\section{Competing interests}

The authors confirm that there are no conflicts of interest that could be perceived as prejudicing the impartiality of the research reported.

\section{Authors' contributions}

MFC conceived of the study, performed the study and coordination and assisted in editing of manuscript. PTC performed the study and drafted the manuscript. MSL conceived of the study and participated in its design and coordination. PYL helped in histology and IHC staining. WCC conceived part of the study and performed the statistical analysis. KDL participated in its design and coordination. All authors read and approved the final manuscript.

\section{Acknowledgements}

The work was support by National Science Council, Taiwan. Grant 98-2314-B182-038-MY2 (to M.F. Chen).

\section{Author details \\ 'Department of Radiation Oncology, Chang Gung Memorial Hospital at Chiayi, Chiayi, Taiwan. ${ }^{2}$ Chang Gung University, College of Medicine, Taoyuan, Taiwan. ${ }^{3}$ Department of Hematology and Oncology, Chang Gung Memorial Hospital at Chiayi, Chiayi, Taiwan. ${ }^{4}$ Graduate Institute of Clinical Medical Sciences, College of Medicine, Chang Gung University, Taoyuan, Taiwan. ${ }^{5}$ Department of Thoracic \& Cardiovascular Surgery, Chang Gung Memorial Hospital, Chiayi, Taiwan. ${ }^{6}$ Department of Pathology, Chang Gung Memorial Hospital at Chiayi, Chiayi, Taiwan.}

Received: 7 November 2012 Accepted: 24 March 2013 Published: 5 April 2013

\section{References}

1. Allum WH, Stenning SP, Bancewicz J, Clark PI, Langley RE: Long-term results of a randomized trial of surgery with or without preoperative chemotherapy in esophageal cancer. J Clin Oncol 2009, 27:5062-7.
2. Sjoquist KM, Burmeister BH, Smithers BM, Zalcberg JR, Simes RJ, Barbour A, et al: Survival after neoadjuvant chemotherapy or chemoradiotherapy for resectable oesophageal carcinoma: an updated meta-analysis. Lancet Oncol 2011, 12:681-92.

3. Pottgen C, Stuschke M: Radiotherapy versus surgery within multimodality protocols for esophageal cancer-a meta-analysis of the randomized trials. Cancer Treat Rev 2012, 38:599-604.

4. Coussens LM, Werb Z: Inflammation and cancer. Nature 2002, 420:860-7.

5. Smyth MJ, Cretney E, Kershaw MH, Hayakawa Y: Cytokines in cancer immunity and immunotherapy. Immunol Rev 2004, 202:275-93.

6. Schafer ZT, Brugge JS: IL-6 involvement in epithelial cancers. J Clin Invest 2007, 117:3660-3.

7. Akira $S$, Taga $T$, Kishimoto $T$ : Interleukin- 6 in biology and medicine. Adv Immunol 1993, 54:1-78.

8. Chen CC, Chen WC, Lu CH, Wang WH, Lin PY, Lee KD, et al: Significance of interleukin- 6 signaling in the resistance of pharyngeal cancer to irradiation and the epidermal growth factor receptor inhibitor. Int J Radiat Oncol Biol Phys 2010, 76:1214-24.

9. Santer FR, Malinowska K, Culig Z, Cavarretta IT: Interleukin-6 transsignalling differentially regulates proliferation, migration, adhesion and maspin expression in human prostate cancer cells. Endocr Relat Cancer 2010, 17:241-53.

10. George DJ, Halabi S, Shepard TF, Sanford B, Vogelzang NJ, Small EJ, et al: The prognostic significance of plasma interleukin- 6 levels in patients with metastatic hormone-refractory prostate cancer: results from cancer and leukemia group B 9480. Clin Cancer Res 2005, 11:1815-20.

11. Oka M, Yamamoto K, Takahashi M, Hakozaki M, Abe T, lizuka N, et al: Relationship between serum levels of interleukin 6 , various disease parameters and malnutrition in patients with esophageal squamous cell carcinoma. Cancer Res 1996, 56:2776-80.

12. Scambia G, Testa U, Benedetti Panici P, Foti E, Martucci R, Gadducci A, et al: Prognostic significance of interleukin 6 serum levels in patients with ovarian cancer. Br J Cancer 1995, 71:354-6.

13. Kim DK, Oh SY, Kwon HC, Lee S, Kwon KA, Kim BG, et al: Clinical significances of preoperative serum interleukin- 6 and C-reactive protein level in operable gastric cancer. BMC Cancer 2009, 9:155.

14. Lukaszewicz-Zajac M, Mroczko B, Kozlowski M, Niklinski J, Laudanski J, Szmitkowski M: Higher importance of interleukin 6 than classic tumor markers (carcinoembryonic antigen and squamous cell cancer antigen) in the diagnosis of esophageal cancer patients. Dis Esophagus 2012, 25:242-9.

15. Rohatgi PR, Swisher SG, Correa AM, Wu TT, Liao Z, Komaki R, et al: Histologic subtypes as determinants of outcome in esophageal carcinoma patients with pathologic complete response after preoperative chemoradiotherapy. Cancer 2006, 106:552-8.

16. Lee CH, Wu DC, Lee JM, Wu IC, Goan YG, Kao EL, et al: Anatomical subsite discrepancy in relation to the impact of the consumption of alcohol, tobacco and betel quid on esophageal cancer. Int J Cancer 2007, 120:1755-62.

17. McConkey DJ, Choi W, Marquis L, Martin F, Williams MB, Shah J, et al: Role of epithelial-to-mesenchymal transition (EMT) in drug sensitivity and metastasis in bladder cancer. Cancer Metastasis Rev 2009, 28:335-44.

18. Bromberg J: Stat proteins and oncogenesis. J Clin Invest 2002, 109:1139-42.

19. Chen MF, Lu MS, Lin PY, Chen PT, Chen WC, Lee KD: The role of DNA methyltransferase $3 \mathrm{~b}$ in esophageal squamous cell carcinoma. Cancer 2012, 118:4074-89.

20. Tartour E, Pere H, Maillere B, Terme M, Merillon N, Taieb J, et al: Angiogenesis and immunity: a bidirectional link potentially relevant for the monitoring of antiangiogenic therapy and the development of novel therapeutic combination with immunotherapy. Cancer Metastasis Rev 2011, 30:83-95.

21. Sharma S, Sharma MC, Sarkar C: Morphology of angiogenesis in human cancer: a conceptual overview, histoprognostic perspective and significance of neoangiogenesis. Histopathology 2005, 46:481-9.

22. Kuonen F, Secondini C, Ruegg C, Molecular Pathways: Emerging pathways mediating growth, invasion and metastasis of tumors progressing in an irradiated microenvironment. Clin Cancer Res 2012, 18:5196-202.

23. Stahl M: Is there any role for surgery in the multidisciplinary treatment of esophageal cancer? Ann Oncol 2010, 21(Suppl 7):vii283-5.

24. Guo Y, Xu F, Lu T, Duan Z, Zhang Z: Interleukin-6 signaling pathway in targeted therapy for cancer. Cancer Treat Rev 2012, 38:904-10. 
25. Siddik ZH: Cisplatin: mode of cytotoxic action and molecular basis of resistance. Oncogene 2003, 22:7265-79.

26. Suchi K, Fujiwara H, Okamura S, Okamura H, Umehara S, Todo M, et al: Overexpression of Interleukin-6 suppresses cisplatin-induced cytotoxicity in esophageal squamous cell carcinoma cells. Anticancer Res 2011, 31:67-75.

27. Trikha M, Corringham R, Klein B, Rossi JF: Targeted anti-interleukin-6 monoclonal antibody therapy for cancer: a review of the rationale and clinical evidence. Clin Cancer Res 2003, 9:4653-65.

28. Leu CM, Wong FH, Chang C, Huang SF, Hu CP: Interleukin-6 acts as an antiapoptotic factor in human esophageal carcinoma cells through the activation of both STAT3 and mitogen-activated protein kinase pathways. Oncogene 2003, 22:7809-18.

29. Chen MF, Lee KD, Lu MS, Chen CC, Hsieh MJ, Liu YH, et al: The predictive role of E2-EPF ubiquitin carrier protein in esophageal squamous cell carcinoma. J Mol Med (Berl) 2009, 87:307-20.

30. Thiery JP: Epithelial-mesenchymal transitions in tumour progression. Nat Rev Cancer 2002, 2:442-54.

31. Gotzmann J, Mikula M, Eger A, Schulte-Hermann R, Foisner R, Beug H, et al: Molecular aspects of epithelial cell plasticity: implications for local tumor invasion and metastasis. Mutat Res 2004, 566:9-20.

32. Bromberg JF, Wrzeszczynska MH, Devgan G, Zhao Y, Pestell RG, Albanese C, et al: Stat3 as an oncogene. Cell 1999, 98:295-303.

33. Levy DE, Darnell JE Jr: Stats: transcriptional control and biological impact. Nat Rev Mol Cell Biol 2002, 3:651-62.

34. Schindler C, Levy DE, Decker T: JAK-STAT signaling: from interferons to cytokines. J Biol Chem 2007, 282:20059-63.

35. Aggarwal BB A, Kunnumakkara AB, Harikumar KB, Gupta SR, Tharakan ST, Koca $C$, et al: Signal transducer and activator of transcription-3, inflammation, and cancer: how intimate is the relationship? Ann N Y Acad Sci 2009, 1171:59-76.

36. Brown JM, Attardi LD: The role of apoptosis in cancer development and treatment response. Nat Rev Cancer 2005, 5:231-7.

37. Olive PL, Banath JP: Phosphorylation of histone $\mathrm{H} 2 \mathrm{AX}$ as a measure of radiosensitivity. Int J Radiat Oncol Biol Phys 2004, 58:331-5.

38. Oshima Y, Yajima S, Yamazaki K, Matsushita K, Tagawa M, Shimada H: Angiogenesis-related factors are molecular targets for diagnosis and treatment of patients with esophageal carcinoma. Ann Thorac Cardiovasc Surg 2010, 16:389-93.

39. Kozin SV, Duda DG, Munn LL, Jain RK: Neovascularization after irradiation: what is the source of newly formed vessels in recurring tumors? J Natl Cancer Inst 2012, 104:899-905.

doi:10.1186/1476-4598-12-26

Cite this article as: Chen et al:: IL-6 expression predicts treatment response and outcome in squamous cell carcinoma of the esophagus. Molecular Cancer 2013 12:26.

\section{Submit your next manuscript to BioMed Central and take full advantage of:}

- Convenient online submission

- Thorough peer review

- No space constraints or color figure charges

- Immediate publication on acceptance

- Inclusion in PubMed, CAS, Scopus and Google Scholar

- Research which is freely available for redistribution

Submit your manuscript at www.biomedcentral.com/submit
C BioMed Central 